\title{
DETERMINING THE MASSES OF WHITE DWARFS IN MAGNETIC CATACLYSMIC VARIABLES FROM X-RAY OBSERVATIONS
}

\author{
KINWAH WU \\ RCfTA, School of Physics \\ University of Sydney, NSW 2006, Australia \\ AND \\ MARK CROPPER AND GAVIN RAMSAY \\ MSSL, University College London \\ Holmbury St. Mary, Surrey, RH5 6NT, United Kingdom
}

\begin{abstract}
.
A method to determine the masses of white dwarfs in magnetic cataclysmic variables from X-ray observations is presented. The method is appropriate for both eclipsing and non-eclipsing systems, for it is insensitive to the orbital inclination of the systems.
\end{abstract}

\section{Introduction}

Magnetic cataclysmic variables (mCVs) are close binaries consisting of a magnetic white dwarf which accretes matter from a Roche-lobe-filling $M$ dwarf companion star. There are two types of mCVs: (i) AM Herculis binaries (AM Hers), in which the white dwarf has a very strong magnetic field that locks all components in the binaries into synchronously rotation and prevents the formation of an accretion disk, and (ii) Intermediate polars (IPs) and DQ Herculis binaries (DQ Hers), which are asynchronously rotating systems with a weaker white dwarf magnetic field only partially disrupting the accretion disk (see Warner 1995). The white dwarf mass is one of the fundamental parameters of $\mathrm{mCVs}$ and has implications for our understanding of the origins, evolution and emission of these binaries.

Most $\mathrm{mCVs}$ are strong X-ray sources, with a keV X-ray spectrum typical of an optically thin bremsstrahlung spectrum. The X-ray emission regions are located near the white dwarf magnetic poles, where the accreting matter 
is heated up by an accretion shock. The accretion shock is formed when the speed of the accreting matter decelerates from supersonic to subsonic near the white dwarf surface. The shock temperature is $T_{s}=3 G \mu m_{H} M_{w} / 8 R_{w} k$, a function of the mass $\left(M_{w}\right)$ and radius $\left(R_{w}\right)$ of the white dwarf only. (Here, $G$ is the gravitational constant, $k$ the Boltzmann constant, $m_{H}$ the mass of hydrogen atom, and $\mu$ the mean molecular mass.) Thus, provided that the shock temperature is measured, the white dwarf mass can be deduced.

By fitting the observed X-ray spectrum with a single-temperature optically thin bremsstrahlung spectrum, it is possible to obtain an effective temperature $T_{e}$ which characterises the emission region. Ishida (1991) assumed that $T_{s} \approx T_{e}$ and, by fitting the GINGA X-ray data, estimated the white dwarf masses of 5 AM Hers and 8 IPs. However, the X-ray emission regions are vertically structured in temperature and density, so that these factors must be taken into account when determining $M_{w}$.

\section{Spectra of X-rays from Structured Emission Regions}

The thickness of the post-shock region $\left(\sim 10^{7} \mathrm{~cm}\right)$ is generally much smaller than the white dwarf radius $\left(\sim 10^{9} \mathrm{~cm}\right)$, so the post-shock accretion flow can be considered as 1-dimensional. The density and temperature structures are therefore determined by a set of 1-D hydrodynamic equations when the appropriate cooling functions are specified. For $\mathrm{mCVs}$, the dominant processes are bremsstrahlung and cyclotron cooling. The treatment of bremsstrahlung cooling is relatively straight-forward because it is optically thin, but the treatment of the optically thick, angle-dependent cyclotron cooling is more complicated. However, for typical parameters of $\mathrm{mCVs}$, the effects of cyclotron cooling can be approximated by a cooling function of power-laws of density and temperature (see Wu, Chanmugam \& Shaviv 1994).

In calculating the X-ray spectra from structured emission regions, we used the MEKAL optically thin plasma code (Mewe, Kaastra \& Liedahl 1995). The absorption of the cooler material in the pre-shock accretion flow was modelled using a modified warm absorber model based on the ABSORI code (Zdziarski \& Magdziarz 1996). The reflection of X-rays from the white dwarf surface was modelled following van Teeseling, Kaastra and Heise (1996). (For the details of the calculations of the model X-ray spectra and the fitting to the observed data, see Cropper, Ramsay \& Wu 1997.) The fit to the GINGA X-ray spectra of the system AM Her at phases of maximum brightness is shown in Figure 1. The white dwarf mass for the best fit is $M_{w}=0.75 M_{\odot}\left(\chi^{2}=0.74,100\right.$ dof $)$. The white dwarf masses of 4 other AM Hers and 8 IPs/DQ Hers obtained by fitting the GINGA data are shown in Table 1. 

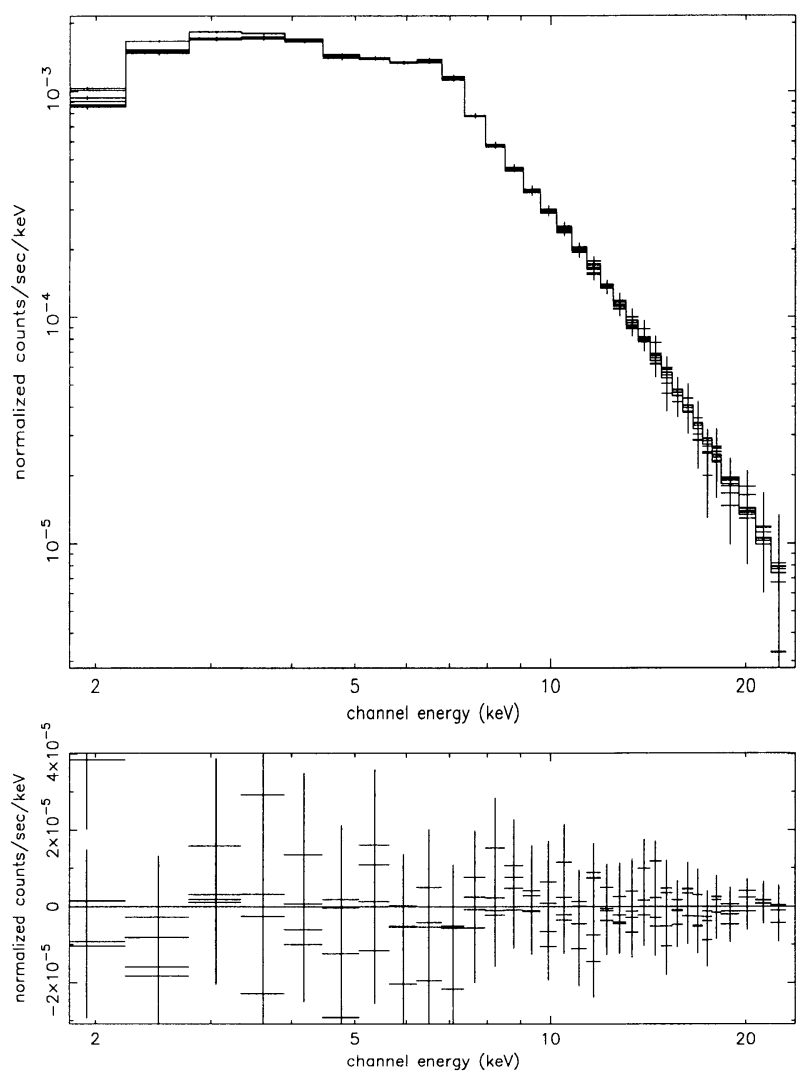

Figure 1. The GINGA data of the system AM Her along with the model fits and residuals.

\section{Discussions}

As shown in Table 1, the mass deduced by Ishida (1991) is systematically lower than the masses obtained in this work. The mass underestimation due to single-temperature fitting can be understood because the local temperatures everywhere in the post-shock region is always lower than $T_{s}$. Thus, the mass estimates of Ishida (1991) are lower limits to the actual values.

Generally, the mass of white dwarfs in mCVs are determined by orbital variations of optical spectral lines emanating from the secondary star. Uncertainties in the orbital inclination of the binary system strongly affects the accuracy with which the mass can be measured using this technique. The method presented here, however, depends only very weakly on the orbital inclination.

The sample we considered is homogeneous, thus it allows a direct com- 
TABLE 1. The white dwarf masses (in $M_{\odot}$ ) deduced from fitting the GINGA X-ray data to our model.

\begin{tabular}{llll}
\hline systems & type & this work & Ishida(1991) \\
\hline AM Her & AM & 0.75 & 0.44 \\
EF Eri & AM & 0.63 & 0.39 \\
BY Cam & AM & 0.70 & 0.63 \\
V834 Cen & AM & 0.49 & 0.33 \\
QQ Vul & AM & 1.03 & 0.66 \\
$\left.<M_{w}\right\rangle_{A M}$ & & $0.72 \pm 0.20$ & $0.49 \pm 0.15$ \\
\hline EX Hya & IP & 0.37 & 0.24 \\
AO Psc & IP & 0.48 & 0.32 \\
FO Aqr & IP & 0.85 & 0.49 \\
TV Col & IP & 1.00 & 0.52 \\
BG CMi & IP & 0.92 & 0.50 \\
PQ Gem & IP & 1.00 & 0.31 \\
TX Col & IP & 0.36 & \\
AE Aqr & DQ & 0.43 & \\
$\left.<M_{w}\right\rangle_{I P / D Q}$ & & $0.68 \pm 0.29$ & $0.40 \pm 0.12$ \\
\hline$\left.<M_{w}\right\rangle_{m C V}$ & & $0.69 \pm 0.25$ & $0.44 \pm 0.14$ \\
\hline
\end{tabular}

parison between the mass distributions of isolated white dwarfs and white dwarfs in binaries. This sheds light on the common-envelope evolution of the binary systems. Our mass determinations can be further improved by refinement of the physical model (e.g. better treatment of cyclotron cooling, two-fluid effects) and by the availability of the X-ray data with higher spectral resolution.

\section{References}

Cropper, M., Ramsay, G. and Wu, K. (1997), MNRAS, in press.

Ishida, M. (1991), PhD Thesis, University of Tokyo, (ISAS RN 505).

Mewe, R., Kaastra, D.S. and Liedahl, D.A. (1995), Legacy (Journal of HEASARC), 6, 16.

van Teeseling, A., Kaastra, J.S. and Heise, J. (1996), $A \& A$, 312, 186.

Warner, B. (1996), Cataclysmic Variable Stars, Cambridge University Press.

Wu, K. Chanmugam, G. and Shaviv, G. (1994), ApJ, 426, 664.

Zdziarski, A.A. and Magdziarz, P. (1996), MNRAS, 279, 21. 